(C)2020, Elsevier. This manuscript version is made available under the CC-BY-NC-ND 4.0 license http:// creativecommons.org/licenses/by-nc-nd/4.0/

\title{
Molecular mechanisms by which SGLT2 inhibitors can induce insulin sensitivity in diabetic milieu: a mechanistic review
}

\author{
Running Title: SGLT2 inhibitors and insulin sensitivity \\ Habib Yaribeygi ${ }^{*}$, Thozhukat Sathyapalan², Mina Maleki, ${ }^{3}$ Tannaz Jamialahmadi, ${ }^{4,5}$ \\ Amirhossein Sahebkar ${ }^{6,7,8^{*}}$
}

\footnotetext{
${ }^{1}$ Research Center of Physiology, Semnan University of Medical Sciences; Semnan, Iran

${ }^{2}$ Academic Diabetes, Endocrinology and Metabolism, Hull York Medical School, University of Hull

${ }^{3}$ Chronic Kidney Disease Research Center, Shahid Beheshti University of Medical Sciences, Tehran, Iran

${ }^{4}$ Halal Research Center of IRI, FDA, Tehran, Iran

${ }^{5}$ Department of Nutrition, Faculty of Medicine, Mashhad University of Medical Sciences, Mashhad, Iran ${ }^{6}$ Biotechnology Research Center, Pharmaceutical Technology Institute, Mashhad University of Medical Sciences, Mashhad, Iran

${ }^{7}$ Neurogenic Inflammation Research Center, Mashhad University of Medical Sciences, Mashhad, Iran

${ }^{8}$ School of Pharmacy, Mashhad University of Medical Sciences, Mashhad,
}

\section{*Corresponding Authors:}

Amirhossein Sahebkar, Biotechnology Research Center, Mashhad University of Medical Sciences, Mashhad 9177948564, Iran. Tel: +985138002299; Fax: +985138002287; E-mail:

sahebkara@mums.ac.ir; amir_saheb2000@yahoo.com

Habib Yaribeygi, Research Center of Physiology, Semnan University of Medical Sciences, Semnan, Iran, Tel: +989355644190 


\begin{abstract}
Sodium-glucose co-transporter-2 inhibitors (SGLT2i) are a relatively newer class of antihyperglycemic medications that reduce blood glucose by inhibition of renal glucose re-uptake, thereby increasing urinary glucose excretion. Although glycosuria is the primary mechanism of action of these agents, there is some evidence suggesting they can reduce insulin resistance and induce peripheral insulin sensitivity. Identifying the molecular mechanisms by which these medications improve glucose homeostasis can help us to develop newer forms of SGLT2i with lesser side effects. We have reviewed the molecular mechanisms and signaling pathways by which SGLT2i therapy improve insulin sensitivity and ameliorates insulin resistance.
\end{abstract}

Keywords: Sodium-glucose co-transporter-2 inhibitors, Insulin Sensitivity, Insulin Resistance, Diabetes Mellitus, Oxidative Stress, Inflammatory Response. 


\section{Introduction}

The global prevalence of diabetes mellitus (DM) is rising rapidly [1]. This chronic disorder results in the development of various debilitating complications as well as deaths worldwide [2]. Chronic hyperglycemia seen in DM can induce various pathophysiologic pathways involved in tissue dysfunction such as oxidative stress, inflammation, apoptosis, fibrosis, hyperexpression of growth factors and hemodynamic variations [3]. Various medications have been developed to normalize hyperglycemia and thereby preventing diabetic complications [4,

5].

Sodium-glucose co-transporter-2 inhibitors (SGLT2i) are a newly developed class of agents that reduce hyperglycemia in DM by inhibition of renal glucose re-uptake and thereby increasing the excretion of glucose in the urine $[5,6]$. These agents act primarily by inducing glycosuria but there is some evidence that they can also induce insulin sensitivity in peripheral tissues [7, 8]. SGLT2i is also associated with some side effects in patients with DM [9-14]. In this review, we have discussed various molecular pathways involved in the modulation of insulin sensitivity by SGLT2i.

Type 2 diabetes (T2DM) is the most prevalent type of diabetes which account for about 9095\% of patients with diabetes and is linked mainly to inadequate response to insulin (decreased insulin sensitivity) and insulin resistance in the peripheral tissues [15-17]. T2DM is a disorder of glucose homeostasis. Glucose is the most abundant monosaccharide in circulation and the preferred substrate for many types of cells in the human body $[18,19]$. This carbohydrate exists mainly as a polysaccharide in starch and then converted by catabolic and enzymatic processes to a hexose monosaccharide $[18,19]$. This monosaccharide is a hydrophilic molecule with a molecular weight of $180.156 \mathrm{~g} / \mathrm{mol}$, which is too large to pass easily across the cell membrane, thereby needing a specific carrier [19]. Glucose has two major ways for entering into the cells: 
(1) via active mechanism by SGLTs and (2) via specific carriers (independent of sodium) [19]. The function of SGLTs is the sections below [20]. Glucose carriers (GLUT) are a family of proteins that provide bidirectional facilitated glucose transport across the cellular plasma membrane [19, 21]. These carriers work without consuming energy and are based on the glucose concentration gradient across the cell membrane. There are at least 14 known GLUT members in human [19, 22]. However, GLUT-1, GLUT-2, GLUT-3 and GLUT-4 are more critical in glucose homeostasis [19]. While GLUT-1 and GLUT-3 are widely expressed, highaffinity and low-capacity transporters, GLUT-2 is high-affinity and low capacity which is expressed in pancreatic beta cells, liver and basolateral side of intestinal and renal tubular cells [19].

Glucose is phosphorylated to glucose-6-phosphate within the beta cells by glucokinase enzyme [23]. This enzyme acts as "glucose sensor" which controls the rate of glucose entering into the islets and in turn controls the rate of insulin secretion [23]. ATP (adenosine triphosphate) synthesis by G6P in beta cells raises the ATP to ADP (adenosine diphosphate) ratio which in turns closes ATP-sensitive $\mathrm{K}^{+}$channels (sensitive to sulfonylureas), depolarizes beta cell membrane and increases intracellular calcium concentration by opening the voltage-dependent $\mathrm{Ca}^{2+}$ channels in beta cells [23]. Rising the levels of intracellular $\mathrm{Ca}^{2+}$ stimulates the exocytosis of secretory granules containing insulin/proinsulin from the beta cells into the circulation [23].

\section{Insulin signal transduction}

Insulin signal transduction (IST) or insulin signaling pathway is initiated by insulin binding to the $\alpha$-subunit of specific receptors known as insulin receptors (IRs), a member of transmembrane tyrosine kinases composed of $\alpha$ and $\beta$ subunits, which are activated by insulin

as well as IGF $^{1} 1$ and 2 [24]. This binding induces structural changes in the $\beta$ subunit by prompting auto-phosphorylation in tyrosine residues followed by downstream events such as

\footnotetext{
${ }^{1}$ Insulin-like growth factor
} 
recruitment of different adaptor proteins i.e. insulin receptor substrates (IRSs), $\mathrm{Shc}^{2}$ protein, and APS protein ${ }^{3}[19,25]$. These processes provide an appropriate binding site for the IRS- $1^{4}$ [25]. Several types of insulin-dependent kinases such as ERK1/2 ${ }^{5}$, atypical $\mathrm{PKC}^{6}, \mathrm{~S} \mathrm{~K} 11^{7}$, $\mathrm{SIK}^{8}{ }^{8}, \mathrm{AKT}, \mathrm{mTOR}^{9}$, and ROCK $1^{10}$ and other types of kinases including $\mathrm{AMPK}^{11}$ and GSK3 $^{12}$ can phosphorylate IRSs to activate them [25, 26]. Activated IRS-1 links to PI3K ${ }^{13}$ and activates it and catalyzes the conversion of $\mathrm{PIP}_{2}{ }^{14}$ to $\mathrm{PIP}_{3}{ }^{15}$ [27]. $\mathrm{PIP}_{3}$ itself is a potent activator for PKB (protein kinase B also known as Akt), which in turn, facilitates glucose entering into the cells by localization of GLUT-4 and inhibits glycogen synthase kinase leading to more glycogen synthesis $[27,28]$. The insulin sensitivity can be modulated in all these steps of insulin signal transduction pathways [29-31].

\section{SGLT2 Inhibitors}

Sodium-glucose co-transporters (SGLT) are two distinct forms of active cotransporters (as type 1 and 2) that mainly localized in the brush border of S2 and S3 segments of proximal renal tubules (as well as in intestines) which reabsorb the filtrated urinary glucose and to prevent glucose excretion [32]. SGLT2 inhibitors (SGLT2i) are a class of medications which specifically inhibit these active carriers and thereby induce urinary glucose excretion leading to lower levels of glycemia in patients with diabetes [5]. This potent oral glucose lowering

\footnotetext{
2 SHC-transforming

${ }^{3}$ adapter protein with a $\mathrm{PH}$ and $\mathrm{SH} 2$ domain

${ }^{4}$ insulin receptor substrate type 1

${ }^{5}$ extracellular signal-regulated kinase 1/2

${ }^{6}$ protein kinase $\mathrm{C}$

${ }^{7}$ Ribosomal protein S6 kinase beta-1

${ }^{8}$ serine/threonine-protein kinase 2

${ }^{9}$ mammalian target of rapamycin

${ }^{10}$ Rho-associated protein kinase 1

${ }^{11}$ AMP-activated protein kinase

12 Glycogen synthase kinase 3

${ }^{13}$ phosphoinositide 3-kinase

${ }^{14}$ Phosphatidylinositol 4,5-bisphosphate

${ }^{15}$ Phosphatidylinositol 3,4,5-trisphosphate
} 
agents work entirely independent of insulin and are dependent on blood glucose levels so that they have a lower risk of hypoglycemia [33].

Since the discovery of phlorizin as the first SGLT2i in the early of 1980s, several forms of these agents have introduced which all reduce the blood glucose below the threshold of tubular transporter for glucose reabsorption [9, 33-35]. However, in addition to its anti-hyperglycemic effects through glycosuria, they may provide other beneficial effects such as suppression of gluconeogenesis, improving insulin sensitivity in peripheral tissues, increasing glucagon response and stimulation of insulin secretion from the beta cells of pancreatic islets [8, 36-38]. Canagliflozin, dapagliflozin, and empagliflozin are the known forms of this class of antidiabetic drugs [39]. The side effects of SGLT2i include dehydration, dizziness, hypotension and genito-urinary infections [39].

\section{GLT2i and iinsulin sensitivity}

Emerging evidence indicates that SGLT2 inhibition can interfere with various steps of IST and modulate insulin sensitivity in peripheral tissues $[7,8,36]$. In the following sections, we present the main involved molecular mechanisms (Table 1).

\section{Inhibition of glucose toxicity}

SGLT2i promotes excretion of glucose through urine, thereby lowering blood glucose and glucotoxicity [9]. Chronic hyperglycemia results in glucotoxicity as seen in the diabetic milieu [40-42]. This toxic milieu negatively affects beta-cell function and is closely related to insulin resistance [43]. On the other hand, strong evidence suggests that SGLT2i-induced glycosuria potentially improves glucotoxicity [44]. 
Mervoric et al. in 2014 has shown that SGLT2 inhibition by dapagliflozin reduces glucotoxicity and thereby improves glycemic control in the diabetic milieu [7]. Two weeks of dapagliflozin therapy in men with diabetes improved glucotoxicity by lowering the fasting blood glucose and increasing tissue sensitivity to insulin [7]. These anti-glucotoxicity effects of SGLT2i are reversible after treatment cessation [7]. List and coworkers in 2011 provided further evidence suggesting that SGLT2i therapy induces insulin sensitivity, beta-cell activity and morphology preservation via ameliorating the glucotoxicity [43]. Kern and colleagues emphasized this finding in 2016, where they found that SGLT2i therapy in diabetic mice improved insulin signaling, leading to better glucose homeostasis [8]. These reports suggest that attenuation of toxic effects of glucose by SGLT2i is one of the main molecular pathways in which they can improve insulin sensitivity [7, 8, 43].

\section{Caloric disposition and weight loss}

Caloric disposition is an established effect of SGLT2i therapy since they promote urinary glucose excretion [45]. Caloric disposition can translate to weight loss as well as a reduction in the circulating lipids [45-47]. It has been demonstrated that SGLT2i therapy can reduce body weight in patients with diabetes [48]. On the other hand, it has been well confirmed that lower mass of adipose tissue and lower levels of circulating lipids are correlated to an increase in insulin sensitivity [49]. The role of inflammatory responses in insulin resistance is completely verified and patients with diabetes have relatively higher levels of circulatory inflammatory mediators [50,51]. The central part of these inflammatory responses is mediated by adipocytes and their related active mediators, known as adipokines [50]. These active biologic adipocytederived cytokines have prominent roles in insulin resistance [52]. There are strong animal and human evidence indicating that high-fat diet and obesity are one of the main underlying causes 
of insulin resistance and the onset of DM $[53,54]$ which SGLT2i therapy can potentially reverse [55]. For example, Shamansurova et al. in 2016, on a diabetic mouse, demonstrated that depletion of adipose tissue improved insulin sensitivity by improving locomotor activity and basal metabolism. Furthermore, increasing adiponectin which is another form of adipocytokines, is associated with insulin sensitivity [56]. Since lesser adipocytes can potentially improve insulin sensitivity, weight loss is strongly recommended in patients with

\section{T2DM [56].}

Xu et al. in 2018 demonstrated that "visceral fat lowering" effect of SGLT2i can markedly improve the insulin sensitivity by ameliorating the lipotoxicity [57]. They reported that SGLT2i therapy in patients with diabetes could attenuate insulin resistance by lowering the rate of adipocyte-induced inflammatory responses by regulating energy homeostasis and a reduction in lipotoxicity-induced insulin resistance [57]. Empagliflozin (SGLT2i) increases insulin sensitivity by ameliorating inflammatory processes and visceral fat lowering via an M2 macrophage polarization-dependent mechanism in white adipose tissue (WAT) of diabetic mice. Also, it promoted browning adipose tissue led to increased energy expenditure and thermogenesis[58]. Xu and colleagues provided evidence for the effects of SGLT2i on WAT browning and fat utilization induced by activating M2 macrophage. Thus, they concluded that empagliflozin has beneficial effects on obesity-related inflammation and insulin resistance. [57]. In a clinical trial with 38 diabetic patients, SGLT2i therapy by canagliflozin significantly improved insulin resistance by a reduction in body weight as well as lowering visceral and ectopic fatty tissues in Japanese patients with T2DM [59]. Furthermore, Singh and coworkers in 2019 provided the same evidence about Indian patients with T2DM suggesting that visceral fat lowering effect of SGLT2i therapy potentially improves insulin sensitivity [60]. Okamoto et al. in 2016 found that dapagliflozin improved adipocyte function which increased adiponectin in obese patients with T2DM [61]. Matsumura and coworkers in 2017 also reported 
that canagliflozin increased adiponectin-induced glucose homeostasis in patients with T2DM [62]. Tobita et al in 2017 demonstrated that canagliflozin therapy in patients with T2DM improved glucose metabolism by reducing visceral fat resulting in metabolic improvement [63]. Garvey et al. in 2018 provided more evidence implying that canagliflozin improved glucose homeostasis by improving lipid metabolism and leptin level in patients with diabetes [64]. This evidence strongly suggests that SGLT2i therapy can increase insulin sensitivity and improve glucose homeostasis at least partly via lowering adipocytes number, fatty tissues and plasma lipids $[57,59,60]$.

\section{Attenuating the inflammatory responses}

Inflammatory responses are potent upstream events in the pathophysiology of DM, contributing to insulin resistance and lowering insulin sensitivity in the peripheral tissues [52]. Patients with diabetes have higher levels of inflammatory mediators, including active cytokines in the blood, which potentially impair IST, thereby reducing insulin sensitivity [52]. Inflammation hypothesis states that inflammation is strongly involved in the pathophysiology of DM $[65,66]$. There is growing evidence that SGLT2i can inhibit or decline inflammatory processes and prevent the expression of inflammatory mediators [67, 68]. It has been suggested that SGLT2 inhibitors can suppress inflammatory responses via both direct (impact on the immune system) and indirect molecular pathways via at least 5 different molecular mechanisms such as modulation of the renin-angiotensin system (RAS), altering hemodynamic changes, reduction in obesity-induced inflammation, modulation of immune system function and readjustment of the redox state in tissue leading to a lower rate of inflammatory responses [57, 69-74]. Since lower inflammatory response can be translated to a reduced impairment in IST and lower risk for insulin resistance, we concluded that anti-inflammatory properties of SGLT2i could be exploited to design novel therapeutic approaches for patients with diabetes. 


\section{Improvement in beta-cell function}

Majority of patients with T2DM have some impairment in pancreatic beta-cell function [75, 76]. They have dysfunctional islets with suboptimal response to stimuli and thereby, inadequate insulin release [76]. The number of functional beta cells is also reduced due to pathophysiological mechanisms such as oxidative damage, fibrosis and inflammation leading to higher rates of apoptotic processes [76, 77]. Improving beta cells function is one of the main targets in patients with diabetes to normalize hyperglycemia [78, 79]. Although one may conclude that SGLT-2i can only increase insulin action, there direct evidences suggesting SGLT2 inhibition increases insulin sensitivity by improving islet cell function in diabetic milieu [55, 80-82]. This action could be possibly through improvement in beta cell-mediated insulin dependent glucose homeostasis.

Jurczak et al. in 2013 demonstrated that SGLT2 deletion in animals with diabetes improves glucose homeostasis and enhances insulin sensitivity by preserving the beta cells function in a diabetic milieu [55]. They have observed a 60\% increase in beta cell mass and lower levels of apoptotic death leading to more functional pancreatic islets in SGLT2 knockout diabetic mice [55]. Kaneto and colleagues in 2017 observed that SGLT2i therapy protects beta cells against glucose toxicity and $\beta$-cell lipotoxicity as well as improves islet cell function leading to increased insulin sensitivity [80]. SGLT2 inhibitory agents can preserve islet function by attenuating the pathophysiologic pathways and deleterious molecular mechanisms [80]. They can suppress glucotoxicity, lipotoxicity, inflammatory processes, fibrotic processes and oxidative damages leading to lower rates of beta cell death and more functional mass of islets thereby improving insulin sensitivity and glucose homeostasis [79-81, 83]. Cheng et al. in 2016 surveyed the potential beneficial effects of empagliflozin and found that it improved glucose homeostasis by preserving the beta-cell function probably via suppressing the glucotoxicity- 
induced oxidative stress-dependent beta-cell death and by increasing regeneration of pancreatic islet cells in diabetic mice [83]. Also, Asahara et al. in 2019 suggested that SGLT2i re-adjust glucose homeostasis by preserving beta cells function [81].

Merovci et al. in 2015 in a human clinical trial demonstrated that SGLT2i with dapagliflozin increased insulin sensitivity by an improvement of beta cells function in T2DM individuals [84]. Al Jobori et al. in 2018 reported that empagliflozin therapy for two weeks is associated with better beta-cell function in patients with diabetes [82]. They showed that empagliflozin therapy increased islets' glucose sensitivity during periods of hyperglycemic clamps which is a method for assessing the function of beta cells [82]. Moreover, Takahara and coworkers in 2015 evaluated the islets function using disposition index and found that four weeks of treatment with ipragliflozin improved beta-cell function in patients with T2DM [85]. These strong experimental and human evidence firmly suggest that SGLT2i therapy improves beta cell function in a diabetic milieu [81-83, 85]. They demonstrated that SGLT2i therapy in diabetic milieu is a potential way for improvement of beta-cell function via several mechanisms leading to an increased number of functional beta-cells and thereby more ability to secrete insulin in response to circulating glucose $[81-83,85]$.

\section{Reduction in oxidative stress}

Free radical generation is a common phenomenon in the biologic milieu which has important biologic functions such as cellular signaling in the physiologic range [86]. In high concentrations, free radical species produces oxidative stress which is one of the main and potent upstream events in various pathophysiologic states including DM [87]. Oxidative stress impairs IST significantly and reduces insulin sensitivity, thereby increasing insulin resistance leading to DM [88, 89]. It negatively affects peripheral insulin sensitivity and down-regulates 
glucose transport system in insulin-dependent cells [88]. Alleviating the free radical production and normalizing its concentration near to the physiologic ranges is one of the main therapeutic targets in DM and preventing its complications [90, 91].

There is growing evidence that SGLT2i improve redox state in the diabetic milieu [92]. They can exert potent antioxidative properties by several molecular mechanisms including improvement in mitochondrial dysfunction $[92,93]$, regulating the RAS (renin-angiotensin system) activity [70, 94], attenuating inflammation-induced free radical generation [95, 96], lowering the AGEs (advanced glycation end products) and AGE/RAGE (receptors for advanced glycation end products) crosstalk [97, 98], down-regulating the pro-oxidant enzymes expression/activity as NADPH (Nicotinamide adenine dinucleotide phosphate) oxidase, eNOS (endothelial nitric oxide synthase) and xanthine oxidase [99, 100]. Based on this evidence, SGLT2 inhibition ameliorates oxidative stress and could improve insulin sensitivity by attenuating oxidative damages in the diabetic milieu [57, 101].

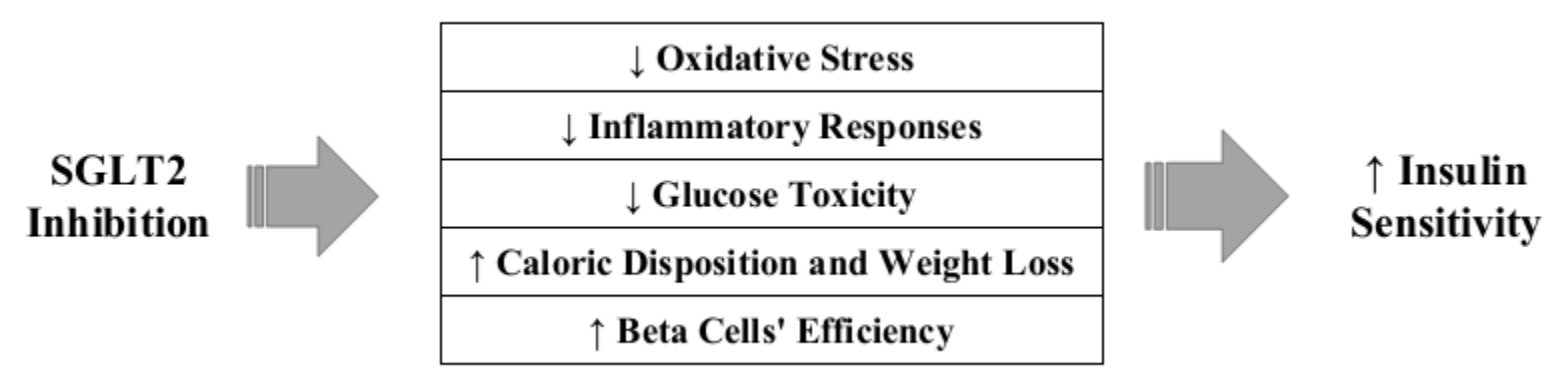

Figure 1: possible pathways by which SGLT2i induce insulin sensitivity 


\section{Conclusion}

Sodium-glucose co-transporter inhibitors are a relatively new class of medications used in the management of diabetes for lowering blood glucose as well as reducing microvascular and macrovascular complications associated with it. Beyond the glycosuric effects, these agents have pleiotropic effects on glucose homeostasis (Table 1). While they are considered as potent glucose-lowering drugs, they can also improve insulin sensitivity and glucose homeostasis via several molecular pathways including reduction of glucotoxicity and lipotoxicity, improvement in beta cell function, reduction of oxidative damages and inflammatory processes as well as induction of caloric disposition and weight loss (Fig 1).

\section{Acknowledgment}

The authors are thankful to the "research center of physiology, Semnan University of medical sciences (Semnan, Iran)" for providing technical support.

\section{Conflict of Interests}

All authors declare that they do not have any conflict of interest in this study.

\section{Funding}

None. 
Table 1. Main molecular mechanisms by which SGLT2i therapy increase insulin sensitivity (IST=insulin signal transduction)

\begin{tabular}{|c|c|c|}
\hline $\begin{array}{c}\text { Molecular } \\
\text { Mechanisms }\end{array}$ & Detailed Effects of SGLT2i & Ref. \\
\hline $\begin{array}{c}\text { Inhibition of Glucose } \\
\text { Toxicity }\end{array}$ & $\begin{array}{c}\text { Normalize hyperglycemia near to physiologic ranges } \\
\text { leading to lowering rates of glucotoxicity and thereby } \\
\text { increasing peripheral insulin sensitivity }\end{array}$ & [7, 8, 43] \\
\hline $\begin{array}{c}\text { Caloric Disposition and } \\
\text { Weight Loss }\end{array}$ & $\begin{array}{c}\text { Induce caloric deposition by glycosuria leading to lesser } \\
\text { adipocytes number as well as lesser adipocytokines and } \\
\text { lipotoxicity thereby improving peripheral insulin } \\
\text { sensitivity }\end{array}$ & [57, 59, 60] \\
\hline $\begin{array}{c}\text { Attenuating the } \\
\text { Inflammatory Responses }\end{array}$ & $\begin{array}{c}\text { Induce peripheral insulin sensitivity by reduction of } \\
\text { various inflammatory processes }\end{array}$ & [58, 68, 70, \\
\hline $\begin{array}{c}\text { Improvement in Beta } \\
\text { Cell Function }\end{array}$ & $\begin{array}{c}\text { Improve beta cell mass/function by ameliorating the } \\
\text { pathophysiological pathways involved in the islets cells' } \\
\text { death }\end{array}$ & {$[55,79-85]$} \\
\hline $\begin{array}{c}\text { Reducing the Oxidative } \\
\text { Stress }\end{array}$ & $\begin{array}{c}\text { Reduce insulin resistance by attenuating the oxidative } \\
\text { stress-dependent IST impairment }\end{array}$ & $\begin{array}{c}\text { [57, 70, 89, 94, } \\
\text { 97, 98, 101] }\end{array}$ \\
\hline
\end{tabular}




\section{References}

1. Mayer-Davis, E.J., et al., Incidence trends of type 1 and type 2 diabetes among youths, 20022012. New England Journal of Medicine, 2017. 376(15): p. 1419-1429.

2. Huang, E.S., et al., Glycemic control, complications, and death in older diabetic patients: the diabetes and aging study. Diabetes care, 2011. 34(6): p. 1329-1336.

3. Forbes, J.M. and M.E. Cooper, Mechanisms of diabetic complications. Physiological reviews, 2013. 93(1): p. 137-188.

4. Chen, Y., F.A. Sloan, and A.P. Yashkin, Adherence to diabetes guidelines for screening, physical activity and medication and onset of complications and death. Journal of Diabetes and its Complications, 2015. 29(8): p. 1228-1233.

5. Association, A.D., Standards of medical care in diabetes-2014. Diabetes care, 2014. 37(Supplement 1): p. S14-S80.

6. Yaribeygi, H., et al., A review of the anti-inflammatory properties of antidiabetic agents providing protective effects against vascular complications in diabetes. Journal of cellular physiology, 2019. 234(6): p. 8286-8294.

7. Merovci, A., et al., Dapagliflozin improves muscle insulin sensitivity but enhances endogenous glucose production. The Journal of clinical investigation, 2014. 124(2): p. 509514.

8. Kern, M., et al., The SGLT2 inhibitor empagliflozin improves insulin sensitivity in $d b / d b$ mice both as monotherapy and in combination with linagliptin. Metabolism, 2016. 65(2): p. 114123.

9. Chao, E.C. and R.R. Henry, SGLT2 inhibition-a novel strategy for diabetes treatment. Nature reviews drug discovery, 2010. 9(7): p. 551.

10. Fadini, G.P. and A. Avogaro, SGLT2 inhibitors and amputations in the US FDA Adverse Event Reporting System. The Lancet Diabetes \& Endocrinology, 2017. 5(9): p. 680-681.

11. Singh, M. and A. Kumar, Risks associated with SGLT2 inhibitors: an overview. Current drug safety, 2018. 13(2): p. 84-91.

12. Scheen, A.J., Does lower limb amputation concern all SGLT2 inhibitors? Nature Reviews Endocrinology, 2018. 14(6): p. 326.

13. Adimadhyam, S., et al., Risk of amputations associated with SGLT2 inhibitors compared to DPP-4 inhibitors: A propensity-matched cohort study. Diabetes, Obesity and Metabolism, 2018. 20(12): p. 2792-2799.

14. Filippas-Ntekouan, S., T.D. Filippatos, and M.S. Elisaf, SGLT2 inhibitors: are they safe? Postgraduate medicine, 2018. 130(1): p. 72-82.

15. Association, A.D., Diagnosis and classification of diabetes mellitus. Diabetes care, 2014. 37(Supplement 1): p. S81-S90.

16. de Faria Maraschin, J., Classification of diabetes, in Diabetes. 2013, Springer. p. 12-19.

17. O'Neal, K.S., J.L. Johnson, and R.L. Panak, Recognizing and appropriately treating latent autoimmune diabetes in adults. Diabetes Spectrum, 2016. 29(4): p. 249-252.

18. Gailliot, M.T. and R.F. Baumeister, The physiology of willpower: Linking blood glucose to selfcontrol, in Self-Regulation and Self-Control. 2018, Routledge. p. 137-180.

19. Hall, J.E., Guyton and Hall textbook of medical physiology e-Book. 2015: Elsevier Health Sciences.

20. Katsiki, N., D. P Mikhailidis, and M. J Theodorakis, Sodium-glucose cotransporter 2 inhibitors (SGLT2i): their role in cardiometabolic risk management. Current pharmaceutical design, 2017. 23(10): p. 1522-1532.

21. Chen, Y. and J. Lippincott-Schwartz, Selective visualization of GLUT4 storage vesicles and associated Rab proteins using IRAP-pHluorin, in Rab GTPases. 2015, Springer. p. 173-179.

22. Huang, S. and M.P. Czech, The GLUT4 glucose transporter. Cell metabolism, 2007. 5(4): p. 237-252. 
23. Rutter, G.A., et al., Pancreatic B-cell identity, glucose sensing and the control of insulin secretion. Biochem J, 2015. 466(2): p. 203-218.

24. Færch, K., et al., Insulin resistance is accompanied by increased fasting glucagon and delayed glucagon suppression in individuals with normal and impaired glucose regulation. Diabetes, 2016: p. db160240.

25. Kiselyov, V.V., et al., Harmonic oscillator model of the insulin and IGF1 receptors' allosteric binding and activation. Molecular systems biology, 2009. 5(1): p. 243.

26. Copps, K. and M. White, Regulation of insulin sensitivity by serine/threonine phosphorylation of insulin receptor substrate proteins IRS1 and IRS2. Diabetologia, 2012. 55(10): p. 25652582.

27. Ho, C.K., G. Sriram, and K.M. Dipple, Insulin sensitivity predictions in individuals with obesity and type Il diabetes mellitus using mathematical model of the insulin signal transduction pathway. Molecular genetics and metabolism, 2016. 119(3): p. 288-292.

28. Koeppen, B.M. and B.A. Stanton, Berne and levy physiology e-book. 2017: Elsevier Health Sciences.

29. Wojtaszewski, J., et al., Insulin signaling and insulin sensitivity after exercise in human skeletal muscle. Diabetes, 2000. 49(3): p. 325-331.

30. Pessin, J.E. and A.R. Saltiel, Signaling pathways in insulin action: molecular targets of insulin resistance. The Journal of clinical investigation, 2000. 106(2): p. 165-169.

31. Chang, L., S.-H. Chiang, and A.R. Saltiel, Insulin signaling and the regulation of glucose transport. Molecular medicine, 2004. 10(7): p. 65.

32. Rajasekeran, H., Y. Lytvyn, and D.Z. Cherney, Sodium-glucose cotransporter 2 inhibition and cardiovascular risk reduction in patients with type 2 diabetes: the emerging role of natriuresis. Kidney international, 2016. 89(3): p. 524-526.

33. Chao, E.C., SGLT-2 inhibitors: a new mechanism for glycemic control. Clinical Diabetes, 2014. 32(1): p. 4-11.

34. Makarova, E., et al., Acute anti-hyperglycaemic effects of an unripe apple preparation containing phlorizin in healthy volunteers: a preliminary study. Journal of the Science of Food and Agriculture, 2015. 95(3): p. 560-568.

35. Clar, C., J.A. Gill, and N. Waugh, Systematic review of SGLT2 receptor inhibitors in dual or triple therapy in type 2 diabetes. BMJ open, 2012. 2(5): p. e001007.

36. Han, S., et al., Dapagliflozin, a selective SGLT2 inhibitor, improves glucose homeostasis in normal and diabetic rats. Diabetes, 2008. 57(6): p. 1723-1729.

37. Wilding, J., et al., Dapagliflozin in patients with type 2 diabetes receiving high doses of insulin: efficacy and safety over 2 years. Diabetes, Obesity and Metabolism, 2014. 16(2): p. 124-136.

38. Ferrannini, E., et al., Metabolic response to sodium-glucose cotransporter 2 inhibition in type 2 diabetic patients. The Journal of clinical investigation, 2014. 124(2): p. 499-508.

39. Reddy, R.M. and S.E. Inzucchi, SGLT2 inhibitors in the management of type 2 diabetes. Endocrine, 2016. 53(2): p. 364-372.

40. Luo, X., et al., Hyperglycemic stress and carbon stress in diabetic glucotoxicity. Aging and disease, 2016. 7(1): p. 90.

41. Campos, C., Chronic hyperglycemia and glucose toxicity: pathology and clinical sequelae. Postgraduate medicine, 2012. 124(6): p. 90-97.

42. Lindmark, S., J. Burén, and J.W. Eriksson, Insulin resistance, endocrine function and adipokines in type 2 diabetes patients at different glycaemic levels: potential impact for glucotoxicity in vivo. Clinical Endocrinology, 2006. 65(3): p. 301-309.

43. List, J.F. and J.M. Whaley, Glucose dynamics and mechanistic implications of SGLT2 inhibitors in animals and humans. Kidney International, 2011. 79: p. S20-S27. 
44. Bertinat, R., F. Nualart, and A.J. Yáñez, SGLT2 inhibitors: glucotoxicity and tumorigenesis downstream the renal proximal tubule? Journal of cellular physiology, 2016. 231(8): p. 16351637.

45. Khat, D.Z. and M. Husain, Molecular mechanisms underlying the cardiovascular benefits of SGLT2i and GLP-1RA. Current diabetes reports, 2018. 18(7): p. 45.

46. Pinto, L.C., et al. Efficacy of SGLT2 inhibitors in glycemic control, weight loss and blood pressure reduction: a systematic review and meta-analysis. in Diabetology \& metabolic syndrome. 2015. BioMed Central.

47. Cai, X., et al., Comparisons of weight changes between sodium-glucose cotransporter 2 inhibitors treatment and glucagon-like peptide-1 analogs treatment in type 2 diabetes patients: A meta-analysis. Journal of diabetes investigation, 2017. 8(4): p. 510-517.

48. Cai, X., et al., The Association Between the Dosage of SGLT2 Inhibitor and Weight Reduction in Type 2 Diabetes Patients: A Meta-Analysis. Obesity, 2018. 26(1): p. 70-80.

49. Clamp, L., et al., Enhanced insulin sensitivity in successful, long-term weight loss maintainers compared with matched controls with no weight loss history. Nutrition \& diabetes, 2017. 7(6): p. e282.

50. Chen, H., Cellular inflammatory responses: novel insights for obesity and insulin resistance. Pharmacological Research, 2006. 53(6): p. 469-477.

51. Rehman, K. and M.S.H. Akash, Mechanisms of inflammatory responses and development of insulin resistance: how are they interlinked? Journal of biomedical science, 2016. 23(1): p. 87.

52. Singer, G. and D.N. Granger, Inflammatory responses underlying the microvascular dysfunction associated with obesity and insulin resistance. Microcirculation, 2007. 14(4-5): p. 375-387.

53. Tan, P., et al., Impact of the prorenin/renin receptor on the development of obesity and associated cardiometabolic risk factors. Obesity, 2014. 22(10): p. 2201-2209.

54. Kahn, S.E., R.L. Hull, and K.M. Utzschneider, Mechanisms linking obesity to insulin resistance and type 2 diabetes. Nature, 2006. 444(7121): p. 840.

55. Jurczak, M.J., et al., SGLT2 deletion improves glucose homeostasis and preserves pancreatic B-cell function. Diabetes, 2011. 60(3): p. 890-898.

56. Shamansurova, Z., et al., Adipose tissue $(P) R R$ regulates insulin sensitivity, fat mass and body weight. Molecular metabolism, 2016. 5(10): p. 959-969.

57. $\mathrm{Xu}, \mathrm{L}$. and T. Ota, Emerging roles of SGLT2 inhibitors in obesity and insulin resistance: Focus on fat browning and macrophage polarization. Adipocyte, 2018. 7(2): p. 121-128.

58. Xu, L., et al., SGLT2 inhibition by empagliflozin promotes fat utilization and browning and attenuates inflammation and insulin resistance by polarizing M2 macrophages in dietinduced obese mice. EBioMedicine, 2017. 20: p. 137-149.

59. Koike, Y., et al., Effect of canagliflozin on the overall clinical state including insulin resistance in Japanese patients with type 2 diabetes mellitus. Diabetes research and clinical practice, 2019. 149: p. 140-146.

60. Singh, A.K., et al., Evidence-Based Consensus on Positioning of SGLT2i in Type 2 Diabetes Mellitus in Indians. Diabetes Therapy, 2019. 10(2): p. 393-428.

61. Okamoto, A., et al., Changes in levels of biomarkers associated with adipocyte function and insulin and glucagon kinetics during treatment with dapagliflozin among obese type 2 diabetes mellitus patients. Drugs in R\&D, 2016. 16(3): p. 255-261.

62. Matsumura, M., et al., Efficacy of additional canagliflozin administration to type 2 diabetes patients receiving insulin therapy: examination of diurnal glycemic patterns using continuous glucose monitoring (CGM). Diabetes Therapy, 2017. 8(4): p. 821-827.

63. Tobita, H., et al., Effects of dapagliflozin on body composition and liver tests in patients with nonalcoholic steatohepatitis associated with type 2 diabetes mellitus: a prospective, openlabel, uncontrolled study. Current Therapeutic Research, 2017. 87: p. 13-19. 
64. Garvey, W.T., et al., Effects of canagliflozin versus glimepiride on adipokines and inflammatory biomarkers in type 2 diabetes. Metabolism, 2018. 85: p. 32-37.

65. Dandona, P., A. Aljada, and A. Bandyopadhyay, Inflammation: the link between insulin resistance, obesity and diabetes. Trends in immunology, 2004. 25(1): p. 4-7.

66. Navarro-González, J.F., et al., Inflammatory molecules and pathways in the pathogenesis of diabetic nephropathy. Nature Reviews Nephrology, 2011. 7(6): p. 327.

67. Nasiri-Ansari, N., et al., Canagliflozin attenuates the progression of atherosclerosis and inflammation process in APOE knockout mice. Cardiovascular diabetology, 2018. 17(1): p. 106.

68. Bonnet, F. and A.J. Scheen, Effects of SGLT2 inhibitors on systemic and tissue low-grade inflammation: The potential contribution to diabetes complications and cardiovascular disease. Diabetes \& metabolism, 2018.

69. Yaribeygi, H., et al., Sodium-glucose cotransporter inhibitors and oxidative stress: An update. J Cell Physiol, 2019. 234(4): p. 3231-3237.

70. Li, L., et al., Effect of a SGLT2 inhibitor on the systemic and intrarenal renin-angiotensin system in subtotally nephrectomized rats. Journal of pharmacological sciences, 2018. 137(2): p. 220-223.

71. Bonora, B.M., et al., Effects of the SGLT2 inhibitor dapagliflozin on cardiac function evaluated by impedance cardiography in patients with type 2 diabetes. Secondary analysis of a randomized placebo-controlled trial. Cardiovascular diabetology, 2019. 18(1): p. 106.

72. Tahara, A., et al., Effects of SGLT2 selective inhibitor ipragliflozin on hyperglycemia, hyperlipidemia, hepatic steatosis, oxidative stress, inflammation, and obesity in type 2 diabetic mice. European journal of pharmacology, 2013. 715(1-3): p. 246-255.

73. Jigheh, Z.A., et al., Empagliflozin alleviates renal inflammation and oxidative stress in streptozotocin-induced diabetic rats partly by repressing HMGB1-TLR4 receptor axis. Iranian journal of basic medical sciences, 2019. 22(4): p. 384.

74. Li, C., et al., SGLT2 inhibition with empagliflozin attenuates myocardial oxidative stress and fibrosis in diabetic mice heart. Cardiovascular diabetology, 2019. 18(1): p. 15.

75. Porte, D. and S.E. Kahn, beta-cell dysfunction and failure in type 2 diabetes: potential mechanisms. Diabetes, 2001. 50(suppl 1): p. S160.

76. Fu, Z., E. R Gilbert, and D. Liu, Regulation of insulin synthesis and secretion and pancreatic Beta-cell dysfunction in diabetes. Current diabetes reviews, 2013. 9(1): p. 25-53.

77. Cooksey, R.C., et al., Oxidative stress, 6-cell apoptosis, and decreased insulin secretory capacity in mouse models of hemochromatosis. Endocrinology, 2004. 145(11): p. 5305-5312.

78. Weng, J., et al., Effect of intensive insulin therapy on B-cell function and glycaemic control in patients with newly diagnosed type 2 diabetes: a multicentre randomised parallel-group trial. The Lancet, 2008. 371(9626): p. 1753-1760.

79. Omar, B., et al., Enhanced beta cell function and anti-inflammatory effect after chronic treatment with the dipeptidyl peptidase-4 inhibitor vildagliptin in an advanced-aged dietinduced obesity mouse model. Diabetologia, 2013. 56(8): p. 1752-1760.

80. Kaneto, H., et al., Beneficial effects of sodium-glucose cotransporter 2 inhibitors for preservation of pancreatic $B$-cell function and reduction of insulin resistance: 钠- 葡萄糖共转 运体2 抑制剂对保护胰腺 6 细胞功能以及降低胰岛素抵抗的有益影响. Journal of diabetes, 2017. 9(3): p. 219-225.

81. Asahara, S.-i. and W. Ogawa, SGLT2 inhibitors and protection against pancreatic beta cell failure. 2019, Springer.

82. Al Jobori, H., et al., Empagliflozin treatment is associated with improved B-cell function in type 2 diabetes mellitus. The Journal of Clinical Endocrinology \& Metabolism, 2018. 103(4): p. $1402-1407$.

83. Cheng, S.T.W., et al., The effects of empagliflozin, an SGLT2 inhibitor, on pancreatic 6 -cell mass and glucose homeostasis in type 1 diabetes. PloS one, 2016. 11(1): p. e0147391. 
84. Merovci, A., et al., Dapagliflozin lowers plasma glucose concentration and improves B-cell function. The Journal of Clinical Endocrinology \& Metabolism, 2015. 100(5): p. 1927-1932.

85. Takahara, M., et al., Ameliorated pancreatic 6 cell dysfunction in type 2 diabetic patients treated with a sodium-glucose cotransporter 2 inhibitor ipragliflozin. Endocrine journal, 2015. 62(1): p. 77-86.

86. Pryor, W., Free radicals in biology. Vol. 6. 2012: Elsevier.

87. Rains, J.L. and S.K. Jain, Oxidative stress, insulin signaling, and diabetes. Free Radical Biology and Medicine, 2011. 50(5): p. 567-575.

88. Tangvarasittichai, S., Oxidative stress, insulin resistance, dyslipidemia and type 2 diabetes mellitus. World journal of diabetes, 2015. 6(3): p. 456.

89. Yaribeygi, H., S.L. Atkin, and A. Sahebkar, A review of the molecular mechanisms of hyperglycemia-induced free radical generation leading to oxidative stress. Journal of cellular physiology, 2019. 234(2): p. 1300-1312.

90. Yaribeygi, $\mathrm{H} .$, et al., Effects of atorvastatin on myocardial oxidative and nitrosative stress in diabetic rats. Comparative Clinical Pathology, 2018. 27(3): p. 691-697.

91. Yaribeygi, H., et al., Antioxidative potential of antidiabetic agents: A possible protective mechanism against vascular complications in diabetic patients. Journal of cellular physiology, 2019. 234(3): p. 2436-2446.

92. Oelze, M., et al., The sodium-glucose co-transporter 2 inhibitor empagliflozin improves diabetes-induced vascular dysfunction in the streptozotocin diabetes rat model by interfering with oxidative stress and glucotoxicity. PLoS One, 2014. 9(11): p. e112394.

93. Sa-nguanmoo, P., et al., SGLT2-inhibitor and DPP-4 inhibitor improve brain function via attenuating mitochondrial dysfunction, insulin resistance, inflammation, and apoptosis in HFD-induced obese rats. Toxicology and applied pharmacology, 2017. 333: p. 43-50.

94. Shin, S.J., et al., Effect of sodium-glucose co-transporter 2 inhibitor, dapagliflozin, on renal renin-angiotensin system in an animal model of type 2 diabetes. PLoS One, 2016. 11(11): $p$. e0165703.

95. Ishibashi, Y., T. Matsui, and S. Yamagishi, Tofogliflozin, a highly selective inhibitor of SGLT2 blocks proinflammatory and proapoptotic effects of glucose overload on proximal tubular cells partly by suppressing oxidative stress generation. Hormone and Metabolic Research, 2016. 48(03): p. 191-195.

96. Wang, X.X., et al., SGLT2 protein expression is increased in human diabetic nephropathy SGLT2 protein inhibition decreases renal lipid accumulation, inflammation, and the development of nephropathy in diabetic mice. Journal of Biological Chemistry, 2017. 292(13): p. 5335-5348.

97. Merhi, Z., et al., Advanced glycation end products upregulate insulin receptor substrate-1 (IRS-1) in granulosa cells. Fertility and Sterility, 2017. 108(3): p. e29.

98. Vlassara, H. and M. Palace, Diabetes and advanced glycation endproducts. Journal of internal medicine, 2002. 251(2): p. 87-101.

99. Kawanami, D., et al., SGLT2 inhibitors as a therapeutic option for diabetic nephropathy. International journal of molecular sciences, 2017. 18(5): p. 1083.

100. Terami, N., et al., Long-term treatment with the sodium glucose cotransporter 2 inhibitor, dapagliflozin, ameliorates glucose homeostasis and diabetic nephropathy in $\mathrm{db} / \mathrm{db}$ mice. PloS one, 2014. 9(6): p. e100777.

101. Osorio, H., et al., Sodium-glucose cotransporter inhibition prevents oxidative stress in the kidney of diabetic rats. Oxidative medicine and cellular longevity, 2012. 2012.

102. Yaribeygi, H., et al., Sodium-glucose cotransporter 2 inhibitors and inflammation in chronic kidney disease: Possible molecular pathways. Journal of cellular physiology, 2019. 234(1): p. 223-230. 
103. Vallon, V., et al., SGLT2 inhibitor empagliflozin reduces renal growth and albuminuria in proportion to hyperglycemia and prevents glomerular hyperfiltration in diabetic Akita mice. American Journal of Physiology-Renal Physiology, 2013. 306(2): p. F194-F204.

104. Takenaka, T., T. Inoue, and Y. Watanabe, How the kidney hyperfiltrates in diabetes: From molecules to hemodynamics. World journal of diabetes, 2015. 6(4): p. 576.

105. Jung, U. and M.-S. Choi, Obesity and its metabolic complications: the role of adipokines and the relationship between obesity, inflammation, insulin resistance, dyslipidemia and nonalcoholic fatty liver disease. International journal of molecular sciences, 2014. 15(4): p. 6184-6223.

106. Jafar, N., H. Edriss, and K. Nugent, The effect of short-term hyperglycemia on the innate immune system. The American journal of the medical sciences, 2016. 351(2): p. 201-211. 